hep-th/9211030

RU-92-40

\title{
Black Hole Remnants and the Information Puzzle
}

\author{
T. BANKS and M. O'LOUGHLIN \\ Dept. of Physics and Astronomy \\ Rutgers University \\ Piscataway, NJ 08855-0849 \\ and \\ ANDREW STROMINGER \\ Dept. of Physics and Astronomy \\ Rutgers University \\ Piscataway, NJ 08855-0849 \\ and \\ Department of Physics \\ University of California \\ Santa Barbara, CA 93106-9530
}

Magnetically charged dilatonic black holes have a perturbatively infinite ground state degeneracy associated with an infinite volume throat region of the geometry. A simple argument based on causality is given that these states do not have a description as ordinary massive particles in a low-energy effective field theory. Pair production of magnetic black holes in a weak magnetic field is estimated in a weakly-coupled semiclassical expansion about an instanton and found to be finite, despite the infinite degeneracy of states. This suggests that these states may store the information apparently lost in black hole scattering processes. 
A semiclassical analysis [1] implies that the radiation emitted by an evaporating black hole is not in a pure state: it is correlated with radiation which falls into the black hole. Causality apparently prevents one from retrieving this information until the black hole is Planck-sized and the semiclassical approximation has broken down [2]. The amount of information that can possibly be retrieved by an external observer (in any reasonable time period) is then limited by energy conservation. There are limits to how much information can be carried out in a finite time by radiation with a finite total energy [3]. One possibility is that the information is simply lost [1]. Another is that the black hole stops evaporating when it reaches the Planck mass, and the information is stored in a stable (or very long lived) remnant (see for example [1,:3]). The remnant must have an essentially infinite number of quantum states in order to accomodate the information. This second possibility has been severely criticized on the grounds that such an infinite degeneracy of remnants would show up as an infinite contribution to real or virtual processes.

A simpler problem, for which an analogous information puzzle occurs, is the scattering of low-energy particles by extremal black holes. This occurs via classical absorption followed by Hawking reemission, and information appears to be lost in the process unless the extremal black hole has an infinite ground state degeneracy.

In fact, it was noted in [4] that recently studied [5,6] extremal magnetic black holes in dilaton gravity appear to have just such an infinite degeneracy associated with an infinite volume throat region of the geometryl. In this paper we will compute the production rate of these states (which will be referred to as "cornucopions" [7]) in a weak magnetic field. For sufficiently weak coupling the rate can be reliably computed in a semiclassical expansion about an instanton describing the creation of a geometry of finite size. We find that the rate is finite, despite the infinite degeneracy. We are able to relate the finite volume of the states produced by tunneling to a horizon which is produced a finite distance down the cornucopion in the process of moving its throat around in the external spacetime. The external field determines both the distance to the Minkowski horizon, and the size of the instanton. This reconciles the euclidean and minkowskian descriptions of the infinite set

1 This infinite degeneracy might be removed by non-perturbative effects [7,8], as will be briefly discussed. In this paper we assume the degeneracy exists and investigate its consequences. 
of cornucopion states. They cannot be treated as elementary particles in either regime, although the classical positions of their throats follow particlelike trajectories.

It was previously argued in [9] that the production rate of magnetically charged black holes in dilaton gravity is finite. There it was suggested that the tunneling process which is responsible for cornucopion production produces an initial state of finite volume which then expands classically to become the essentially infinite cornucopion volume. As in the inflationary universe, the number of states of the final geometry that can be produced in this way is limited by the size of the initial geometry. The present paper confirms the final conclusions of [9], but the physical picture developed here is quite different than that envisaged there.

While our results do not directly bear on the information puzzle for neutral black holes, they certainly suggest that the standard arguments against remnants may be wrong in the neutral case as well. In the conclusion we will argue that the cornucopion hypothesis is really a hybrid between the hypotheses of remnants and information loss, which avoids the difficulties of both.

Four-dimensional dilaton-Maxwell gravity is described by the action

$$
S=\frac{1}{16 \pi} \int d^{4} x \sqrt{-g} e^{-2 \phi}\left(R+4(\nabla \phi)^{2}-\frac{1}{2} F^{2}\right) .
$$

This theory has a three-parameter family of magnetic black hole solutions labelled by the mass $M$, magnetic charge $Q$ and asymptotic value $\phi_{0}$ of the dilaton [5, 6]. For $M^{2}$ greater than the critical value $\frac{1}{4} Q^{2}$, the causal structure of these black holes is similar to that of the Schwarzschild geometry, with a singularity behind an event horizon. For $M^{2}<\frac{1}{4} Q^{2}$, the solutions resemble $M^{2}<Q^{2}$ Reissner-Nordstrom: there is a naked singularity at the origin.

The nature of the extremal $M^{2}=\frac{1}{4} Q^{2}$ solutions is, however, dramatically affected by the presence of the dilaton [6]. The extremal solution is given by

$$
\begin{aligned}
d s^{2} & =-d t^{2}+\left(1+\frac{Q}{y}\right)^{2}\left(d y^{2}+y^{2} d^{2} \Omega\right), \\
e^{2\left(\phi-\phi_{0}\right)} & =1+\frac{Q}{y}, \\
F & =Q \epsilon
\end{aligned}
$$




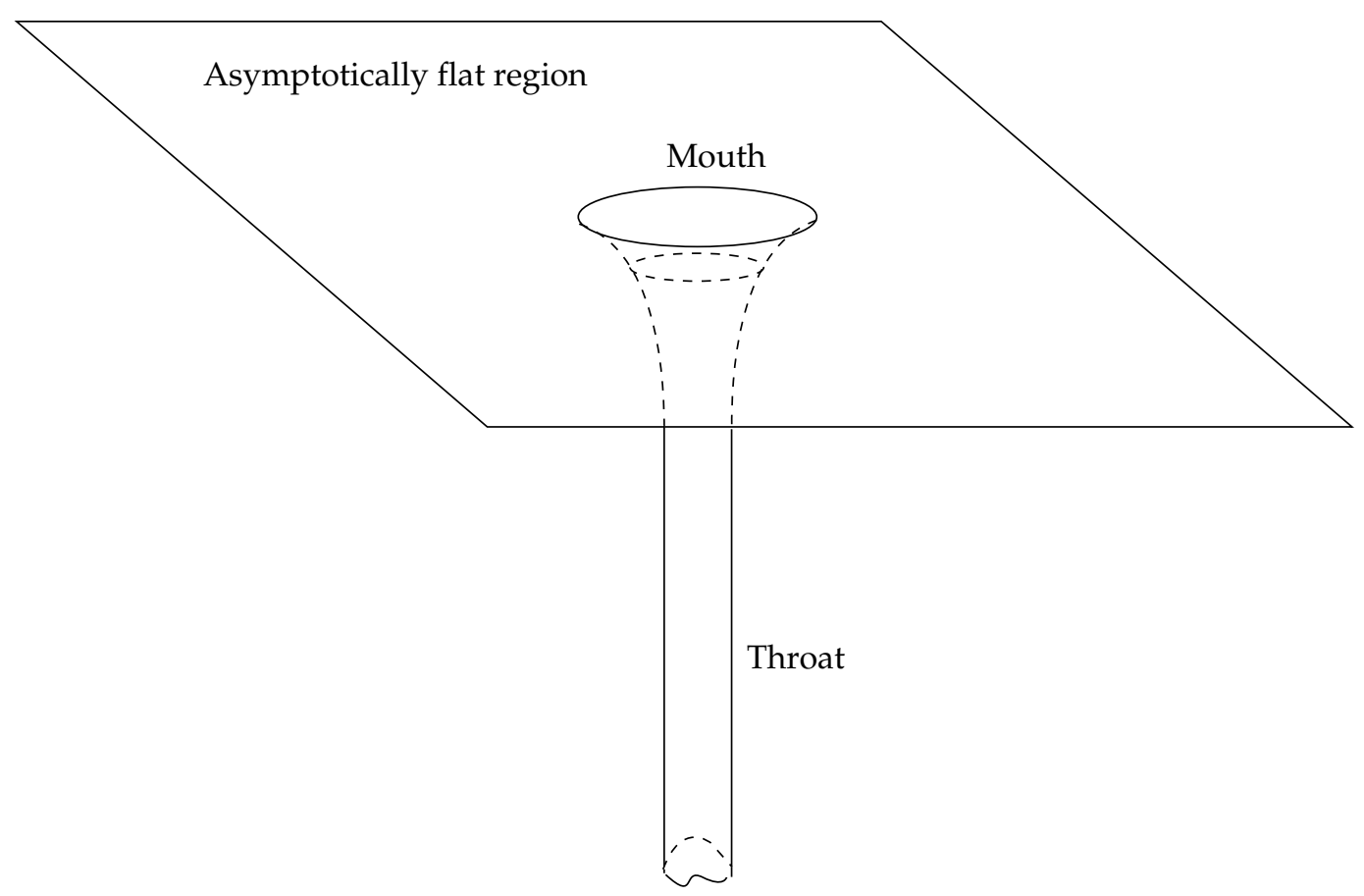

Fig. 1: The spatial geometry of an extremal dilatonic magnetic black hole. The cross sections of the throat are two spheres.

where $\epsilon$ is the volume form on the two sphere of constant radius, normalized so that its integral is equal to $4 \pi$. As illustrated in Figure 1, the spatial geometry is asymptotically flat for large $y$, but as $y$ approaches zero, there is a semi-infinite "throat" whose cross sections are two spheres of constant radius $Q$. The region $y \sim Q$, where the throat begins, will be referred to as the mouth. The extremal geometry is geodesically complete, and there are no horizons or singularities. However there are extra null infinities, since light rays can travel forever down the throat. The Penrose diagram is depicted in Figure 2. The dilaton field grows linearly in this region, which means that gravity becomes strongly coupled far down the throat.

Recent attention has focused on the problem of low-energy $S$-wave scattering of massless scalars or charged fermions by this extremal black hole [4]. As soon as any energy is thrown into the throat, a horizon develops and the black hole becomes non-extremal. It then presumably returns to its extremal ground state via Hawking emission. At energies much less than $\frac{1}{Q}$, this process can be analyzed with the two-dimensional effective field theory which governs low-energy dynamics in the throat region. Regarding this region as 


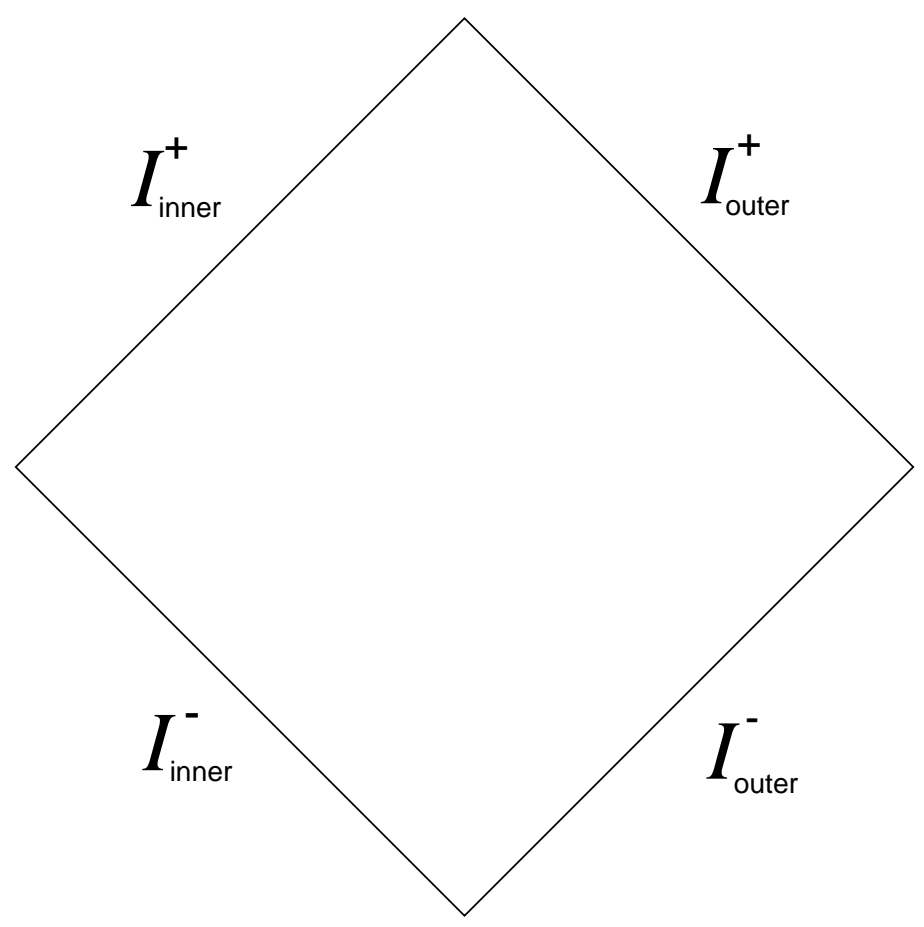

Fig. 2: Penrose diagram of an extremal magnetic dilatonic black hole.

a compactification from four to two dimensions with a two sphere threaded by magnetic flux, the two-dimensional effective field theory is given by 2

$$
S_{2}=\frac{Q^{2}}{2 \pi} \int d^{2} x \sqrt{-g} e^{-2 \phi}\left(R+4(\nabla \phi)^{2}+\frac{1}{Q^{2}}-\frac{1}{2} G^{A} G^{A}\right)
$$

together with the appropriate matter action. $G^{A}, A=1,2,3$ are $S U(2)$ gauge field strengths arising from the isometries of the two sphere. Although mentioned in [10], these modes have been ignored in previous work because rotational invariance prevents them from being excited in $S$-wave scattering. They will however play a major role in the rotationally non-invariant processes considered later in this paper. We are suppressing in (3) the relic of the original gauge field $F$ as it will not enter into later considerations. The theory (3) has a vacuum solution

$$
\begin{aligned}
g_{a b} & =\eta_{a b}, \\
\phi & =-\frac{x}{2 Q},
\end{aligned}
$$

2 The overall factor of $Q^{2}$, arising from the volume of the two spheres, was absorbed into a shift of $\phi$ in [7, 10]. $Q$-independent factors have been absorbed into a shift of $\phi$ in (3). 
which corresponds to the extremal four-dimensional magnetic black hole. There are also two-dimensional black hole solutions [11] which correspond to non-extremal fourdimensional magnetic black holes. Stationary solutions with non-zero $G$ correspond to the charged rotating black hole solutions of [12]. Four-dimensional particle-hole scattering corresponds to two dimensional black hole formation/evaporation.

In [4] semiclassical equations describing this process for a large number $N$ of matter fields were derived. It was conjectured that it is described by a unitary $S$-matrix, with the infinite amount of information about the incoming scattering state being stored in an infinite number of zero-energy bound states of the extremal black hole (i.e. cornucopions). The possibility of an infinite degeneracy of states arises because of the infinite volume of the throat region. This conjecture was shown to be false for large $N$ in [7] and [8]. Rather a singularity is formed and the approximation breaks down. However in [13] evidence was presented that, after accounting for the effects of Fadeev-Popov ghosts, the conjecture of [4] might be realized when $N<24$.

We shall henceforth assume that, at least in some model, particle-hole scattering is indeed unitary and the information is accounted for by the infinite set of cornucopions. Whether or not this is the case is an important question that is not the subject of this paper. Rather, we wish to question the following argument that any remnant scenario of this type is in fact experimentally ruled out.

In a low-energy effective field theory, the argument goes, these degenerate cornucopions are described by an effective action

$$
S_{e f f}=\sum_{i=1}^{\infty} \int d^{4} x \sqrt{-g} e^{-2 \phi}\left(\mathcal{D}_{\mu} \Psi_{i} \mathcal{D}^{\mu} \Psi_{i}+\frac{Q^{2}}{4} \Psi_{i}^{2}\right),
$$

where $\Psi_{i}$ creates the $i$ th cornucopion; and $\mathcal{D}$ is the (somwhat complicated) covariant derivative for a magnetically charged particle. The low energy field theory contains instantons, depicted in Figure 3, describing cornucopion pair production in a weak magnetic field. It is usually asserted that as long as the magnetic field is small compared to the cornucopion mass $M=Q / 2$, and the Schwinger length $M / Q B$ much larger than the diameter of the throat, these instantons should give an accurate estimate of the production rate. According to Schwinger, the production rate (with our conventions) is proportional 
to

$$
\exp \left[\frac{-\pi Q e^{-2 \phi_{0}}}{4 B}\right]
$$

for each species of cornucopion. $e^{2 \phi_{0}}$ plays the role of $\hbar$ in the exponent. For $Q$ of order one (in Planck units) and ordinary magnetic fields, (6) is a fantastically small number. However we must sum over all species of cornucopions, which yields an infinite total production rate! Since cornucopion production has not been observed, one concludes that there cannot possibily be enough states of an extremal black hole to account for all the potential information loss in scattering experiments.

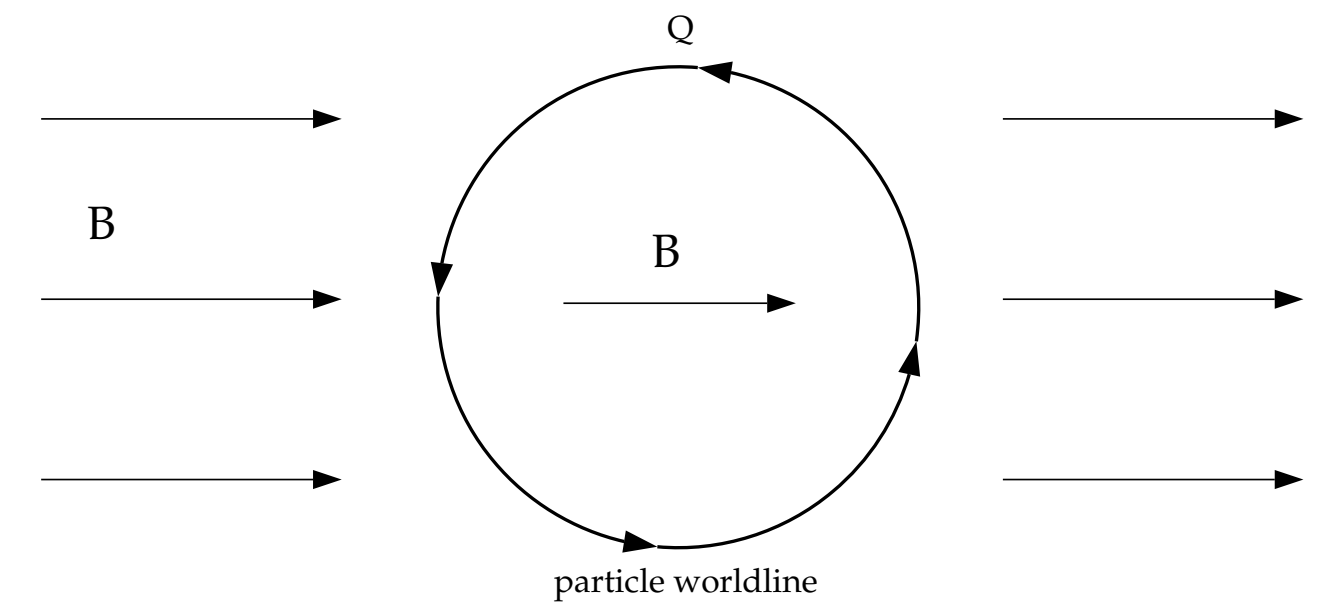

Fig. 3: The instanton for pair production of charged particles in a magnetic field is a circular Euclidean orbit.

This argument is false. In fact cornucopions can not be simply described by an effective action of the type (5). To sharpen this discussion, it is useful to consider a theory in which the throat has a finite, but very long length, say a million light years 3 . There will then be a large but finite, say $\mathcal{N} \sim e^{10^{6} \times 10^{7} \times 10^{42}}$, number of distinguishable cornucopions.

Now let us suppose we are given a box containing $10 \mathcal{N}$ cornucopions and asked to sort them according to species into $\mathcal{N}$ boxes. Assuming they are described by an effective

3 Such a theory can be constructed by replacing the gauge field in (四) with a GUT theory broken to $U(1)$. For a low GUT scale, the theory has 'tHooft-Polyakov monopoles with small gravitational corrections. For a GUT scale greater than the Planck scale, the magnetically charged solutions are identical to (2). By tuning the GUT scale to the approach the Planck scale from below, one obtains an arbitrarily long throat terminated at a monopole. 
action of the type (5), and given a large array of very well-equipped experimentalists, this is a straightforward task. One simply performs interference experiments to determine which cornucopions are identical and then drops them in the boxes.

This experiment is doomed to failure if it is performed in less than a million years. The reason is simple. If we have fully determined the species of the extremal black hole, we have obtained information about the quantum state in a region of spacetime a million light years away. Causality clearly must prevent us from obtaining this information. We emphasize that what we have described is a true violation of causality, and not an EinsteinPodolsky-Rosen paradox. The operation of moving the cornucopions around within a room in a matter of minutes or hours can be described by the action of local operators in a region of spacetime that is causally separated from the bottom of the throat during the same time interval. If our experiment allowed us to determine whether two states of the cornucopion localized at the bottom of the throat were the same or not, these operators would not commute with a local operator which changed the state near the bottom of the throat during the same time interval. We conclude that (5) can not provide a full description of the low-energy dynamics of cornucopions; and we accordingly have no right to conclude that the cornucopion production rate diverges.

What goes wrong when we actually try to do the experiment? In order to interfere two cornucopions, we must move them. Some energy will necessarily go down the throat, and a horizon will form. This is the second law of black hole mechanics: interactions with a black hole increase the area of the horizon. The more gently the cornucopions are moved, the farther down the throat the horizon is formed. If they are moved extremely gingerly (on a time scale exceeding a million years) a horizon may not form at all - but this is not possible for experiments which take less than a million years. Once a horizon forms all bets are off. Presumably it eventually recedes via Hawking emission, but the state of the cornucopions is greatly perturbed by the attempt to measure it. Even if they were initially in identical states the probability that they remain so after the scattering is completed is very small. The formation of horizons in the internal geometry prevents us from deciding whether the cornucopions are bosons, fermions or distinguishable particles as long as the length and time scales involved in the scattering process are short compared 
to the cornucopion length. If this length is finite, a naive effective field theory analysis would be valid only at much longer length scales.

These observations can be quantified in the context of the pair production of magnetic black holes in a weak magnetic field. For the Einstein-Maxwell theory without dilatons, this production rate was computed using instanton methods in [14]. The instanton -in analogy to that discovered by Schwinger for point particles - is the analytic continuation of a real time configuration describing two constantly accelerating, oppositely charged dilatonic magnetic black holes in a weak magnetic field. As depicted in Figure 4, along the hyperbolic wordlines of the two black hole mouths are attached two "fins" corresponding to the throats of the black holes. We depict these fins as having finite length because the application of an external force on the extremal black hole produces a horizon a finite distance down the throat 1 . The geometry of the uniformly accelerating cornucopions has a timelike Killing field which generates motion along the hyperbolae and is null (zero norm) at the horizons.

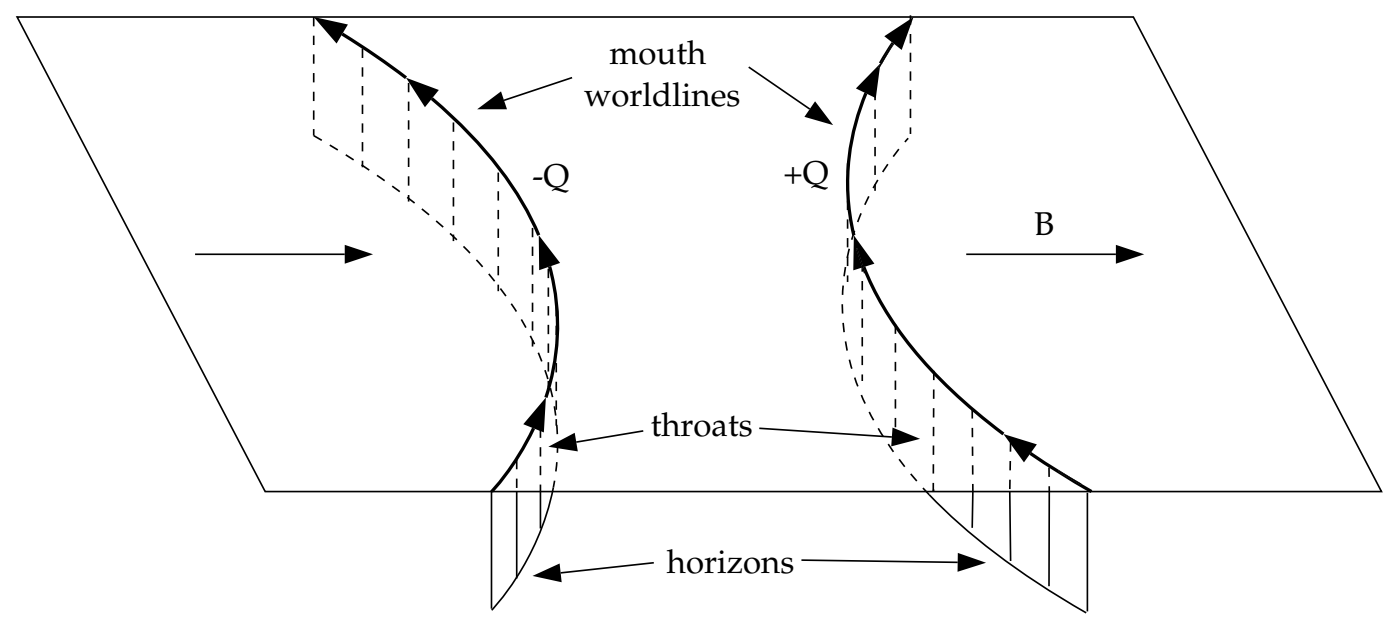

Fig. 4: Uniform acceleration of two oppositely charged black holes in a magnetic field $\mathrm{B}$. The throats are terminated at the horizon.

The analytically continued euclidean geometry is depicted in Fig. 5. The hyperbolae become circles, and the Killing field generates a rotational symmetry. This symmetry has

4 The formation of a horizon may also be understood as a requirement for the black hole to thermally equillibrate with the acceleration radiation. 
a fixed point on the horizons, which collapse to a single point, closing up the end of the fins. Thus, as in previous examples of gravitation instantons, the euclidean section is a continuation of only the region of the Minkowski geometry outside the horizon. The result is that the euclidean continuation of the throat region has the shape of a cup which is joined onto the asymptotically flat region along its rim, the worldline of the black hole mouth.

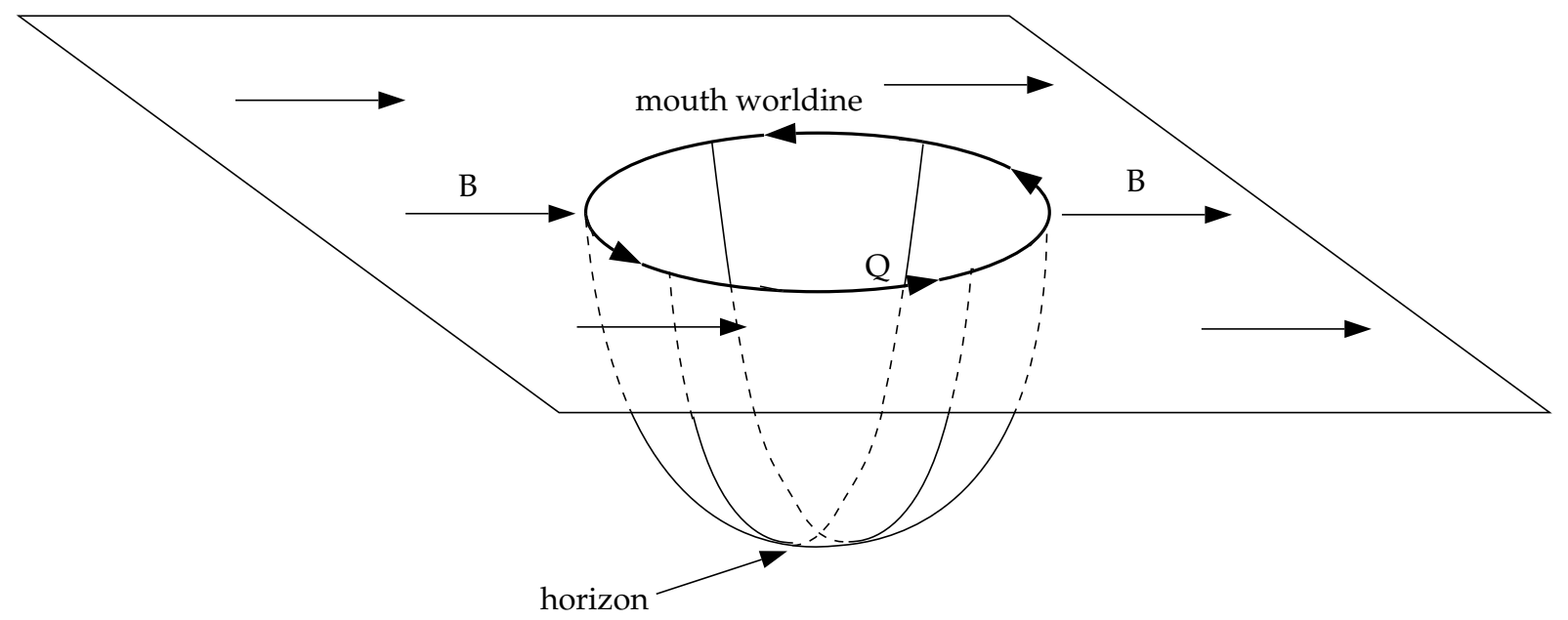

Fig. 5: Instanton for magnetic pair creation of charged black holes. A finite volume cup-shaped region is attached to the asymptotic region along the circular mouth worldline.

The exact instanton is known for Einstein-Maxwell theory [14]. It is certainly difficult, but perhaps possible, to construct the analogous solution in the dilaton-modified theory. However at scales below $\frac{1}{Q}$ one may use the novel effective field theory described in «4, 7 , 10 to describe the instanton. This effective field theory has mixed dimensionality. There is a four dimensional part in which the black holes are described as charged point particles moving in weak magnetic, gravitional and dilatonic fields. We shall consider only magnetic

5 In our effective field theory the two-spherical cross section of the throat is replaced by a point, and the world surface of this infinitely thin throat is the surface of the cup. We also note that the solutions of the field equations have negative curvature, so that Fig. 5, and the term cup may be somewhat misleading. We have not been able to find graphics software capable of drawing a faithful image of the geometry on a sheet of paper. 
fields, so the relevant part of the four-dimensional action is (for a single black hole)

$$
S_{4}=\frac{1}{32 \pi} e^{-2 \phi_{0}} \int d^{4} x F^{2}+\frac{1}{2} e^{-2 \phi_{0}} Q \int d \tau \tilde{A}_{\mu}(X) \dot{X}^{\mu}+e^{-2 \phi_{0}} m \int d \tau \sqrt{\dot{X}^{\mu} \dot{X}_{\mu}},
$$

where $X^{\mu}(\tau)$ is the world line of the black hole mouth and - because the charge is magnetic - $d \tilde{A}$ is the dual of the field strength.

In the effective field theory, the mouth worldline is attached to a two-dimensional fin of possibly infinite depth. The dynamics of the fin are governed by the two-dimensional dilaton gravity action (3). In addition there are interaction terms involving products of two and four-dimensional operators integrated along the mouth worldline. These allow energy and information exchange between the four and two-dimensional regions. In general one expects every interaction allowed by symmetries to appear. One such relevant term is ( in a non-relativistic approximation, valid for small $B^{A}$ )

$$
\frac{i \gamma}{\pi} e^{-2 \phi_{0}} Q \int H^{A}(0, \tau) B^{A}(X(\tau))
$$

where $B^{A}$ is the four-dimensional magnetic field, $H^{A}(0, \tau)$ is the two-dimensional $\mathrm{SU}(2)$ one-form potential at the boundary of the two-dimensional region. We have not calculated $\gamma$ but expect that it is order one. (8) is gauge invariant because $B^{A}$ carries $\mathrm{SU}(2)$ charge, i.e. it transforms under rotation. (8) implies that an external magnetic field will excite one of the $\mathrm{SU}(2)$ fields, and that if $H^{A}$ is non-zero on the boundary of the two-dimensional region, the black hole has a magnetic dipole moment.

We now describe the instanton for black hole pair production in the language of this effective field theory. The four-dimensional portion consists of a weak magnetic field in euclidean $R^{4}$ with the mouth worldline moving in a circular orbit of radius $\mathrm{R}$. As noted above, the two-dimensional portion is a cup whose rim is joined to the $R^{4}$ along the mouth worldline. The geometry of this cup is a truncation of the extremal, charged euclidean black hole solution discussed in [15]:

$$
\begin{aligned}
d s^{2} & =Q^{2}\left[\frac{\sinh ^{2} r}{(\cosh \alpha+\cosh r)^{2}} d \tau^{2}+d r^{2}\right], \\
e^{-2 \phi} & =e^{-2 \phi_{0}} \frac{(\cosh \alpha+\cosh r)}{\left(\cosh \alpha+\cosh r_{M}\right)}, \\
G & =\frac{i \epsilon_{2} \sinh \alpha}{Q(\cosh \alpha+\cosh r)},
\end{aligned}
$$


where

$$
\begin{aligned}
& 0<\tau<2 \pi(\cosh \alpha+1), \\
& 0<r<r_{M},
\end{aligned}
$$

( $\epsilon_{2}$ is the volume form on two space), and $r_{M}$ obeys

$$
R=\frac{Q \sinh r_{M}(\cosh \alpha+1)}{\left(\cosh \alpha+\cosh r_{M}\right)}
$$

insuring that the boundary length is $2 \pi R$. $G$ is an abelian field strength in a subgroup of $S U(2)$ (determined by the external $B$ field) and $\alpha$ parameterizes its magnitude.

The parameter $\alpha$ is determined by matching conditions as follows. The boundary conditions on the two-dimensional field theory (see [10] for a detailed discussion) require that $G$ vanish if the operator insertion (8) is regarded as just inside the boundary. Thus the conserved black hole charge

$$
q=-\left.* e^{-2 \phi} G\right|_{r_{M}},
$$

(* is the Hodge dual) must equal minus the charge $\frac{B}{Q}$ near the boundary:

$$
q=-\frac{i e^{-2 \phi_{0}} \sinh \alpha}{Q\left(\cosh \alpha+\cosh r_{M}\right)}=-\frac{i \gamma B e^{-2 \phi_{0}}}{Q} .
$$

We are interested in large $R,($ small $B)$, which implies large $r_{M}$. One then has

$$
\sinh \alpha \approx \gamma B \cosh r_{M}
$$

or using (11)

$$
\begin{aligned}
\cosh \alpha & \approx \frac{R}{Q}, \\
\sinh r_{M} & \approx \frac{R}{\gamma Q B} .
\end{aligned}
$$

Note that the correct euclidean $S U(2)$ field strength is imaginary, as appropriate for instantons which describe tunneling to configurations with non-zero electric fields [16]. The external magnetic field carries real Minkowski angular momentum, so its effect on the twodimensional Euclidean action is to insert a Wilson loop on the boundary of the cup. The semiclassical field due to a Wilson loop is always imaginary. 
It remains to extremize the entire four plus two-dimensional action with respect to $R$. The two-dimensional action contributes only a surface term along the rim of the cup, which can be absorbed (for small $B$ ) by a renormalization of the mass in (7) . It is easily seen that small $B$ implies large $R$ and the problem reduces to the one analyzed by Schwinger. One finds that

$$
R=\frac{1}{2 B}
$$

The action is

$$
I=-\frac{\pi Q e^{-2 \phi_{0}}}{4 B}+\mathcal{O}\left(B^{0}\right)
$$

and the production rate is finite and proportional to

$$
\exp \left[-\frac{\pi Q}{4 B} e^{-2 \phi_{0}}\right]
$$

To go beyond this leading result, one should compute the one-loop determinant for whatever quantum fields are present in the theory. This will include a factor that counts the number of states in the black hole throat. However, since the throat is cut off at a finite distance from the mouth, only a finite number of states will be counted. Loosely speaking, there is no pair production of states whose excitations are localized far down the throat.

Note that our computation actually describes the production of a Wheeler wormhole rather than an actual pair of monopoles of opposite charge located inside the throats of growing cornucopions (the picture proposed in [9]). We believe that this is the correct picture of cornucopion production even in a theory which contains real monopoles, if the monopoles are sufficiently heavy so that the throat length of the static extremal black hole is much longer than the distance to the horizon. Experiments done over the time scale of the motion of the cornucopion mouth cannot probe the depths of the throat and discover whether or not there are actually monopoles there. They cannot distinguish between a Wheeler wormhole and a monopole pair. Our instanton was contructed by analytic continuation of these Minkowski trajectories and must therefore conserve magnetic flux without the intervention of monopoles. Of course, ordinary monopole pair creation inside the wormhole can still occur, and it can evolve into the sort of geometry suggested in [9]. 
Let us remind the reader of the limitations on our semiclassical analysis. Our considerations are valid only so long as the horizon forms in a region in which the coupling is still weak. The coupling at the horizon is given by $e^{2 \phi_{H}}=e^{2 \phi_{0}} / \gamma B$. Thus, if we make the external field extremely weak, we must also decrease the asymptotic value of the dilaton in order to stay in the weak coupling regime everywhere in spacetime.

In summary, the rate of cornucopion pair production in a weak magnetic field has been estimated in a weakly coupled semiclassical expansion and found to be finite, despite naive expectations to the contrary. Thus an infinite quantum degeneracy of extremal black hole states may well provide a resolution to the information puzzle for particle-hole scattering.

While we believe "infinite volume" remnants of the general type discussed here may store the information lost in particle-hole scattering without implying excessive pair production, it should be stressed that there is little practical difference between this type of information storage and actual information loss. Cornucopions resolve the information puzzle by forming a new asymptotic region of spacetime. The $S$-matrix of the observer in the original asymptotic region of space (i.e. the region which existed before cornucopion formation) is not in itself unitary. If the charged black hole settles down into a nonsingular, horizon-free static spacetime, as we have been assuming in this paper, then the asymptotic observer could in principle construct (two-dimensional!) detectors and send them down the cornucopion throat to help him verify the unitarity of the total $S$-matrix. Even this will not suffice to extract all information from the black hole if the information is receding down the throat at the speed of light. This will certainly be the case if the stationary end point of black hole evaporation resembles the asymptotically DeSitter solutions of the $N<24$ semiclassical equations discussed in [13.

Thus, in general, our resolution of the information paradox is in a sense a realization of Hawking's proposal that information is lost in black hole evaporation. Note however that this occurs in the context of a completely unitary quantum mechanical evolution (at least at the level of the semiclassical approximation for the metric). Spacetimes like that discussed in 13 can be foliated with a complete set of Cauchy surfaces, and quantum field theory in such backgrounds is unitary. The new feature that allows for information loss is the creation of a new future asymptotic region of space, causally disconnected from the 
region that existed before the formation of the black hole. The local evolution operator is unitary but the $S$-matrix of the observer in the initial asymptotic region is not.

It is clear from this discussion that, contrary to Hawking's original suggestion, virtual processes do not lead to information loss. Virtual cornucopion formation and evaporation corresponds to a history in the path integral in which a finite volume spatial slice of cornucopion is temporarily formed and then relaxes back to the vacuum. No new asymptotic region is formed and no loss of information occurs. An effective lagrangian taking into account the effect of small virtual fluctuations in the geometry will obey the rules of quantum mechanics, and the difficulties of Hawking's proposal pointed out in [17] are avoided. Our picture of black hole evaporation thus contains Remnants (of arbitrary states of large black holes) Without Remnants (being produced copiously in the laboratory or in virtual loops) and Information Loss (for asymptotic observers) Without Information Loss (in virtual processes).

Could the scenario proposed here be a general resolution of the information loss paradox, valid for all processes involving black holes? We are not sure. In the present context, conservation of magnetic flux prevents the throat of the black hole from pinching off, creating a disjoint universe into which information can be truly lost. It is not obvious what would prevent such a process in the case of a neutral black hole. 


\section{Acknowledgements}

We are grateful to A. Dabholkar, S. Giddings, G. Horowitz, J. Preskill, N. Seiberg and S. Shenker for useful conversations. A. S. would like to thank the Rutgers Theory Group for their hospitality. This work was supported in part by DOE Grants, 91ER40618 and DE-FG-60590-ER-40559. 


\section{References}

[1] S. W. Hawking, Commun. Math. Phys. 43 (1975) 199 ;

Phys. Rev. D14 (1976) 2460.

[2] See for example, R.M. Wald, "Black holes, singularities, and predictability," in Quantum Theory of Gravity, S.M. Christensen ed. (Adam Hilger, Bristol U.K. 1984), or J. Preskill, "Do black holes destroy information?" Caltech preprint CALT-68-1805, hep-th/9209058.

[3] Y. Aharonov, A. Casher and S. Nussinov, Phys. Lett. 191B (1987) 51.

[4] C.G. Callan, S.B. Giddings, J.A. Harvey, and A. Strominger, "Evanescent black holes," Phys. Rev. D45 (1992) R1005.

[5] G. Gibbons and K. Maeda, Nucl. Phys. B298 (1988) 741.

[6] D. Garfinkle, G. Horowitz, and A. Strominger, Phys. Rev. D43 (1991) 3140; Erratum: Phys. Rev. D45 (1992) 3888.

[7] T. Banks, A. Dabholkar, M.R. Douglas, and M. O'Loughlin, "Are horned particles the climax of Hawking evaporation?" Phys. Rev. D45 (1992) 3607.

[8] J.G. Russo, L. Susskind, and L. Thorlacius, "Black hole evaporation in 1+1 dimensions" Phys. Lett. B292 (1992) 13.

[9] T. Banks and M. O'Loughlin, "Classical and quantum production of cornucopions at energies below $10^{18} \mathrm{GeV}$ ", Rutgers preprint RU-92-14 (1992).

[10] S. B. Giddings and A. Strominger, "Dynamics of extremal black holes", Phys. Rev. D46 (1992) 627.

[11] E. Witten, "On string theory and black holes," Phys. Rev D44 (1991) 314.

[12] A.Sen, "Rotating charged black hole solution in heterotic string theory", Phys. Rev. Lett. 69 (1992) 1006, J. Horne and G. Horowitz, "Rotating dilaton black holes", Phys. Rev. D46 (1992) 1340.

[13] A. Strominger, "Fadeev-Popov ghosts and 1+1 dimensional black hole evaporation," Phys. Rev. D, to appear, hep-th /9205028.

[14] D. Garfinkle, A. Strominger, "Semiclassical Wheeler wormhole production" Phys. Lett. B256 (1991) 246.

[15] M. McGuigan, C. Nappi and S. Yost, "Charged black-holes in two-dimensional string theory", Nucl. Phys.B375 421 (1992); G.W. Gibbons and M.J. Perry, "The physics of 2d stringy spacetime", preprint hep-th/9204090 (1992); C. Nappi and A. Pasquinucci, "Thermodynamics of two-dimensional black-holes", gr-qc/9208002 (1992).

[16] K. Lee, "Wormholes and Goldstone bosons" Phys. Rev. Lett. 61 (1988) 263

[17] T. Banks, M. Peskin, L. Susskind,"Difficulties for the evolution of pure states into mixed states", Nucl. Phys. B244 (1984) 125. 